\title{
Adaptive Risk Hedging for Call Options under Cox-Ingersoll-Ross Interest Rates
}

\author{
Niloofar Ghorbani, Andrzej Korzeniowski \\ Department of Mathematics, University of Texas at Arlington, Arlington, USA \\ Email: niloofar.ghorbani@mavs.uta.edu,korzeniowski@uta.edu
}

How to cite this paper: Ghorbani, N. and Korzeniowski, A. (2020) Adaptive Risk Hedging for Call Options under Cox-IngersollRoss Interest Rates. Journal of Mathematical Finance, 10, 697-704.

https://doi.org/10.4236/jmf.2020.104040

Received: November 2, 2020

Accepted: November 22, 2020

Published: November 25, 2020

Copyright $\odot 2020$ by author(s) and Scientific Research Publishing Inc. This work is licensed under the Creative Commons Attribution International License (CC BY 4.0).

http://creativecommons.org/licenses/by/4.0/

\begin{abstract}
We present a solution to the problem posed by Zhang et al. [1] regarding Call Option price $C_{T}$ under linear investment hedging for the stochastic interest rate modeled by a CIR Process. A closed form representation for $C_{T}$ by expected value of the path-integral along a square functional of $n$-dimensional Ornstein-Uhlenbeck process is derived. The method is suitable for Monte-Carlo simulation and illustrated by an example.
\end{abstract}

\section{Keywords}

European Call Option, Linear Stock Investment Strategy, Cox-Ingersoll-Ross Model, Ornstein-Uhlenbeck Process, Numeraire and Martingale Measure

\section{Introduction}

In recent years, thanks to steady growth of financial derivatives market, various generalizations of the classical option pricing model were developed. Namely, a combination of stochastic interest rates along with dynamic investing strategies in the underlying security prior to option expiration has been proposed for the purpose of hedging the investment risks. It turned out that selling a security proportionally to its dropping price for Put Option and buying the security proportionally to its rising price for Call Option (both under European Black -Scholes Model) resulted in lower Option price as shown by Wang and Wang [2] [3]. Zhang et al. [1] extended the result for Call Option to stochastic interest rates following the Vasicek model and asked whether Call Option price can be established for stochastic interest rates under the Cox-Ingersoll-Ross (CIR) model [4]. An extensive background and the literature on the subject can be found in [1].

The main obstacle in solving the problem for CIR is the fact that the closed form solution to the stochastic differential equation (SDE) is no longer available 
(in general), unlike in Vasicek interest rate given explicitly by the gaussian process.

This paper is concerned with the derivation of the Call Option price for the linear investment under CIR interest rate. A key benefit of CIR process is that in some economies the interest rates always stay positive, and consequently the Vasicek model is not applicable due to allowing the interest rates to become negative. In what follows we adopt the model setup and notation from [1].

European Call Option under the linear investment strategy triggers stock buying whenever the stock price exceeds the strike price. The investment fraction is defined by:

$$
Q(S)=\left\{\begin{array}{lc}
0 & S \leq K \\
\frac{\beta}{\alpha K}(S-K) & K \leq S \leq(1+\alpha) K \\
\beta & S \geq(1+\alpha) K
\end{array}\right.
$$

where

$S$ is stock price.

$Q(S)$ is the stock investment proportion, which is equal to the value of the stock investment divided by $A$, where $A$ is the entire investment amount.

$K$ is strike price of the option.

$\alpha$ is the investment strategy index, indicating the stock investment occurs during the period in which the stock price increases from $K$ to $(1+\alpha) K$.

$\beta$ is the maximum value of the stock investment proportion.

It was found in [1] that the Call Option value $V_{T}$ based on the linear investment with parameters $\alpha, \beta$, strike price $K$, and the terminal stock price $S_{T}$ reads as follows:

$$
V_{T}= \begin{cases}0 & S_{T} \leq K \\ \left(1+\frac{\beta}{\alpha}\right)\left(S_{T}-K\right)-\frac{\beta S_{T}}{\alpha} \ln \left(\frac{S_{T}}{K}\right) & K \leq S_{T} \leq(1+\alpha) \\ S_{T}-K-\frac{\beta S_{T}}{\alpha} \ln (1+\alpha)+K \beta & S_{T}>(1+\alpha) K\end{cases}
$$

We will use the above formula for the stock price that satisfies SDE with drift depending on the random interest rate, whose SDE follows CIR.

\section{The Market Model}

\section{Notations}

Consider the stock price $S_{t}$ dynamics

$$
\mathrm{d} S_{t}=r_{t} S_{t} \mathrm{~d} t+\sigma_{1} S_{t} \mathrm{~d} W_{1, t}, \quad S(0)=S_{0}>0,0 \leq t \leq T .
$$

By Ito lemma, the stock price at time $T$ can be expressed as

$$
S_{T}=S_{0} e^{\int_{0}^{T}\left(r_{s}-\frac{\sigma_{1}^{2}}{2}\right) d s+\int_{0}^{T} \sigma_{1} \mathrm{~d} W_{1, t}} .
$$


Furthermore, consider the interest rate $r_{t}$ dynamics known as Cox-IngersollRoss model

$$
\mathrm{d} r_{t}=a\left(b-r_{t}\right) \mathrm{d} t+\sigma_{2} \sqrt{r_{t}} \mathrm{~d} W_{2, t}, \quad r(0)=r_{0}>0, \quad 0 \leq t \leq T
$$

where $W_{1, t}$ and $W_{2, t}$ are independent Brownian motions on the probability space $\left(\Omega, \mathcal{F},\left\{\mathcal{F}_{t}\right\}_{t \geq 0}, P\right)$ adapted to the filtration $\mathcal{F}_{t}$.

The definition and lemma below are standard.

Definition 2.1.1. [5] [6] A numeraire is any strictly positive $\mathcal{F}_{t}$-adapted stochastic process $N_{t}$ that can be taken as a unit of reference when pricing an asset $X_{t}$ as follows

$$
\hat{X}_{t}=\frac{X_{t}}{N_{t}} .
$$

Lemma 2.1.1. [5] [6] Assume there exists a numeraire $N$ and the corresponding probability measure $Q_{N}$. Then the price of any traded asset (without intermediate payments) $X$ relative to $N$ is a martingale under $Q_{N}$

$$
E^{Q_{N}}\left[\frac{X_{T}}{N_{T}} \mid \mathcal{F}_{t}\right]=\frac{X_{t}}{N_{t}}, \quad 0 \leq t \leq T
$$

In this paper we consider the money market account $B_{t}=\mathrm{e}^{\int_{0}^{t} r_{s} \mathrm{ds}}$ with the stochastic interest rate $r_{t}$ as numeraire. The measure associated with this numeraire is a risk-neutral measure denoted by $Q$ and by the lemma reads

$$
E^{Q}\left[\frac{X_{T}}{B_{T}} \mid \mathcal{F}_{t}\right]=\frac{X_{t}}{B_{t}}, \quad 0 \leq t \leq T
$$

The derivative price is then obtained by calculating the conditional expectation of its terminal payoff

$$
H_{0}=E_{Q}\left[\mathrm{e}^{-\int_{0}^{T} r_{s} \mathrm{ds}} H_{T} \mid \mathcal{F}_{0}\right]
$$

where $H_{T}$ is the derivative's payoff at time $T$. The filtration $\mathcal{F}_{0}$ does not have an effect on calculation of the expectation and Formula (2.7) can be written as

$$
H_{0}=E_{Q}\left[\mathrm{e}^{-\int_{0}^{T} r_{s} \mathrm{ds}} H_{T}\right] .
$$

Indeed, the option price $C$ at initial time discounted by the money market account numeraire under the risk-neutral measure is represented by

$$
C=E_{Q}\left[\mathrm{e}^{-\int_{0}^{T} r_{s} \mathrm{ds}} V_{T}\right]
$$

where $V_{T}$ is the Call Option payoff at maturity time

$$
V_{T}=h\left(S_{T}\right)=h\left(S_{0} \mathrm{e}^{\int_{0}^{T}\left(r_{\mathrm{s}}-\frac{\sigma_{1}^{2}}{2}\right) \mathrm{ds}+\int_{0}^{T} \sigma_{1} \mathrm{~d} W_{1, t}}\right),
$$

with

$$
h(x)=\max [x-K, 0] \geq 0,
$$


and $V_{T}$ previously defined by (1.2).

\section{CIR Model via Ornstein-Uhlenbeck Process}

Cox-Ingersoll-Ross (1985) introduced a square-root term in the diffusion coefficient of the Vasicek model which brings a solution to the positivity problem encountered in Vasicek model. It is well-known that in general there is no closed-form solution to the CIR model Equation (2.3). However, it turns out that in some cases one can obtain the closed form solution in terms of the OrnsteinUhlenbeck (OU) process. For the sake of completeness, we state and verify this fact in the following lemma.

Lemma 3.1. [7] Consider the $n$-dimensional OU process

$$
\mathrm{d} X_{t}^{i}=-\alpha X_{t}^{i} \mathrm{~d} t+\sigma \mathrm{d} W_{t}^{i}
$$

where $W_{t}^{i}$ are $n$ independent Brownian motions, $i=1, \cdots, n$. Let

$$
Y_{t}=\sum_{i=1}^{n}\left(X_{t}^{i}\right)^{2} .
$$

Note that

$$
\begin{aligned}
\mathrm{d}\left(X_{t}^{i}\right)^{2} & =2 X_{t}^{i} \mathrm{~d} X_{t}^{i}+2 \mathrm{~d}\left\langle X^{i}\right\rangle_{t} \\
& =\left(-2 \alpha\left(X_{t}^{i}\right)^{2}+\sigma^{2}\right) \mathrm{d} t+2 \sigma X_{t}^{i} \mathrm{~d} W_{t}^{i}
\end{aligned}
$$

Thus

$$
\begin{aligned}
\mathrm{d} Y_{t} & =\mathrm{d}\left(\sum_{i=1}^{n}\left(X_{t}^{i}\right)^{2}\right)=\sum_{i=1}^{n} \mathrm{~d}\left(X_{t}^{i}\right)^{2} \\
& =\left(-2 \alpha Y_{t}+n \sigma^{2}\right) \mathrm{d} t+2 \sigma \sum_{i=1}^{n} X_{t}^{i} \mathrm{~d} W_{t}^{i},
\end{aligned}
$$

where the second step follows from the independence of the Brownian motions. Next note that the process

$$
Z_{t}=\int_{0}^{t} \sum_{i=1}^{n} X_{u}^{i} \mathrm{~d} W_{u}^{i}
$$

is a martingale with quadratic variation

$$
\langle Z\rangle_{t}=\int_{0}^{t} \sum_{i=1}^{n}\left(X_{u}^{i}\right)^{2} \mathrm{~d} u=\int_{0}^{t} Y_{u} \mathrm{~d} u .
$$

Consequently, by Levy's characterization theorem, the process

$$
\tilde{W}_{t}=\int_{0}^{t} \frac{1}{\sqrt{Y_{u}}} \sum_{i=1}^{n} X_{u}^{i} \mathrm{~d} W_{u}^{i}
$$

is a Brownian motion. Therefore

$$
\mathrm{d} Y_{t}=\left(-2 \alpha Y_{t}+n \sigma^{2}\right) \mathrm{d} t+2 \sigma \sqrt{Y_{t}} \mathrm{~d} \tilde{W}_{t}
$$

whereas

$$
\mathrm{d} r_{t}=a\left(b-r_{t}\right) \mathrm{d} t+\sigma_{2} \sqrt{r_{t}} \mathrm{~d} W_{2, t}
$$


Direct comparison $\left(Y_{t} \equiv r_{t}\right)$ yields

$$
a=2 \alpha, \quad b=\frac{n \sigma^{2}}{a}=\frac{n \sigma^{2}}{2 \alpha} \text { and } \sigma_{2}=2 \sigma .
$$

To solve (3.1) multiply by $X_{t} \mathrm{e}^{\alpha t}$ to have

$$
\mathrm{d}\left(X_{t}^{i} \mathrm{e}^{\alpha t}\right)=\mathrm{e}^{\alpha t} \mathrm{~d} X_{t}^{i}+\alpha \mathrm{e}^{\alpha t} X_{t}^{i} \mathrm{~d} t=\sigma \mathrm{e}^{\alpha t} \mathrm{~d} W_{t}^{i}
$$

which upon integration from 0 to $t$ gives

$$
X_{t}^{i}=\mathrm{e}^{-\alpha t} X_{t}^{i}(0)+\int_{0}^{t} \sigma \mathrm{e}^{-\alpha(t-s)} \mathrm{d} W_{s}^{i} .
$$

Notice that (3.2)-(3.3) imply that $r_{t}$ has non-central chi square distribution.

The parameter a corresponds to the speed of adjustment to the mean $b$, and $\sigma_{2}$ is the short rate volatility. The drift $a\left(b-r_{t}\right)$ is exactly the same as in Vasicek model, however, the volatility in CIR model is $\sqrt{r_{t}} \sigma_{2}$ as opposed to $\sigma_{2}$ for Vasicek. The drift ensures mean reversion of the interest rate towards the long run value $b$, with the speed of adjustment governed by the strictly positive parameter $a$. To ensure that interest rate $r_{t}$ stays positive for all $t$ we must assume $2 a b>\sigma_{2}^{2}$ in equation (2.3) which in turn requires $n \geq 3$ in (3.2).

It is worth noting that in the Vasicek model Zhang et al. [1] utilized a zero-coupon bond as numeraire, which lead to the option price under the forward measure. This approach entails to drift change in the SDE for the interest rate. This method, when applied to the CIR model, would require extension of our Lemma to OU process with variable drift, and ultimately would have introduced more complexity to the closed form representation of the interest rate. As a result, our representation was derived under the risk neutral measure, which is more suitable for Monte Carlo simulation.

\section{Call Option Price under CIR Model}

Fact

Stock price $S_{T}$ under CIR model reads (2.2). We rewrite this expression as follows

$$
\begin{gathered}
S_{T}=S_{0} \mathrm{e}^{\int_{0}^{T} r_{s} \mathrm{~d} s-\int_{0}^{T} \frac{\sigma_{1}^{2}}{2} \mathrm{~d} s+\int_{0}^{T} \sigma_{1} \mathrm{~d} W_{1, t}} \\
S_{T}=S_{0} \mathrm{e}^{R+C+Z}
\end{gathered}
$$

where

$$
\begin{gathered}
R=\int_{0}^{T} r_{s} \mathrm{~d} s \\
C=-\int_{0}^{T} \frac{\sigma_{1}^{2}}{2} \mathrm{~d} s \\
Z=\int_{0}^{T} \sigma_{1} \mathrm{~d} W_{1, t} \sim N\left(0, \sigma_{1}^{2} T\right)
\end{gathered}
$$

with independent random variables $R, Z$.

Remark 4.1.1. Even though $r_{t}$ has known non-central chi-square distribution (by (3.2), $Y_{t} \equiv r_{t}$ ), the distribution of its integral $\int_{0}^{t} r_{s} \mathrm{~d} s$ is unknown (unlike 
gaussian with known mean and variance in the Vasicek case) and thus not suitable for direct calculations. Nevertheless, the path integral $\int_{0}^{t} r_{s} \mathrm{~d} s$ leads to straightforward Monte Carlo simulation, thanks to squared OU process representation of $r_{t}$

Theorem 4.1.1. Based on the notation established in (4.1)-(4.2), the explicit form of $V_{T}$ reads

$$
V_{T}=\left\{\begin{array}{l}
0, R+Z \leq \ln \left(\frac{K}{S_{0}}\right)+\frac{\sigma_{1}^{2}}{2} T \\
\left(1+\frac{\beta}{\alpha}\right)\left(S_{0} \mathrm{e}^{-\frac{\sigma_{1}^{2}}{2} T+R+Z}-K\right)-\frac{\beta}{\alpha} S_{0} \mathrm{e}^{-\frac{\sigma_{1}^{2}}{2} T+R+Z}\left(\ln S_{0}-\frac{\sigma_{1}^{2}}{2} T+R+Z-\ln K\right), \ln \frac{K}{S_{0}}+\frac{\sigma_{1}^{2}}{2} T \leq R+Z \leq \ln \frac{K(1+\alpha)}{S_{0}}+\frac{\sigma_{1}^{2}}{2} T \\
S_{0} \mathrm{e}^{-\frac{\sigma_{1}^{2}}{2} T+R+Z}-K-\frac{\beta}{\alpha}\left(S_{0} \mathrm{e}^{-\frac{\sigma_{1}^{2}}{2} T+R+Z}\right)(\ln (1+\alpha))+K \beta, R+Z>\ln \frac{K(1+\alpha)}{S_{0}}+\frac{\sigma_{1}^{2}}{2} T
\end{array}\right.
$$

Even though the distribution of $S_{T}$ is unknown, (2.10) can still be calculated by Monte Carlo simulation thanks to the path integral representation of $S_{T}$.

\section{Monte Carlo Simulation}

\subsection{Discretization}

In order to do simulation and use the theorem (4.1.1) we need to simulate the stock price $S_{T}$ as expressed by (4.1)-(4.2). The only part that requires attention is the path integral $R=\int_{0}^{t} r_{t} \mathrm{~d} t$. To calculate $R$ we implement Riemann approximation with the discretization on $[0, T]$ as follows:

$$
r_{t}=\sum_{i=1}^{n}\left(X_{t}^{i}\right)^{2}=\sum_{i=1}^{n}\left[\mathrm{e}^{-\alpha t} X_{t}^{i}(0)+\int_{0}^{t} \sigma \mathrm{e}^{-\alpha(t-s)} \mathrm{d} W_{s}^{i}\right]^{2} .
$$

We have

$$
\begin{aligned}
\int_{0}^{T} r_{t} \mathrm{~d} t & =\int_{0}^{T} \sum_{i=1}^{n}\left(X_{t}^{i}\right)^{2} \mathrm{~d} t=\sum_{i=1}^{n} \int_{0}^{T}\left(X_{t}^{i}\right)^{2} \mathrm{~d} t \\
& \approx \sum_{i=1}^{n}\left[\sum_{l=1}^{m} h\left[\mathrm{e}^{-\alpha t} X_{t}^{i}(0)+\sum_{k=1}^{l} \sigma \mathrm{e}^{-\alpha h l-\alpha h k}\left(W_{h k}^{i}-W_{h(k-1)}^{i}\right)\right]^{2}\right]
\end{aligned}
$$

with $m=\frac{T}{h}$ for the time step size $h$.

\subsection{Example}

We illustrate our method by simulating the Black-Scholes European Call under CIR interest rates for six months, one year and a two year Leap $(T=0.5,1,2)$. We chose $h=0.01$ and number of trials $N=10,000$, for accuracy of the Brownian Motion approximation and simulation respectively. The results are listed in the table.

\section{Simulation Parameters:}

Investment Indexes $\alpha=0.2$ and $\beta=0.5$. 
Table 1. Estimated value of Call Option price.

\begin{tabular}{cccc}
\hline Call Option price $C_{T}$ & \multicolumn{3}{c}{ Terminal time $T$} \\
\cline { 2 - 4 } & 0.5 & 1 & 2 \\
\hline CIR with Investment Strategy & 2.25 & 2.26 & 2.22 \\
CIR without Investment Strategy & 3.42 & 3.32 & 3.27 \\
\hline
\end{tabular}

Stock volatility $\sigma_{1}=0.5$.

Interest rate volatility $\sigma_{2}=0.2$.

CIR model parameters $a=1, b=0.02$.

The initial stock price $S_{0}=40$.

The strike price $K=45$.

As expected, by Table 1, the option price with investment under CIR is smaller than the Option price without investment and shows that the Linear Investment hedging lowers the investment risk for the Call Option holder.

\section{Conclusion}

We present an effective way for calculating Call Option price in the case of randomly evolving interest rates for the Cox-Ingersoll-Ross model. The method uses Monte Carlo simulation of interest rates path integrals, which is readily carried out thanks to OU process representation. Furthermore, our approach can be extended to any other stochastic interest rate model with suitable solution representation (e.g. some transformation of Brownian Motion) of its underlying SDE.

\section{Conflicts of Interest}

The authors declare no conflicts of interest regarding the publication of this paper.

\section{References}

[1] Zhang, X., Shu, H.S., Kan, X., Fang, Y.Y. and Zheng, Z.W. (2018) The Call Option Pricing Based on Investment Strategy with Stochastic Interest Rate. Journal of Mathematical Finance, 8, 43-57. https://doi.org/10.4236/jmf.2018.81004

[2] Wang, X.F. and Wang, L. (2007) Study on the Black-Scholes Stock Put Option Model Based on Dynamic Investment Strategy. International Journal of Innovative Computing, Information and Control, 3, 1755-1780.

[3] Wang, X.F. and Wang, L. (2009) Study on Black-Scholes Option Pricing Model Based on General Linear Investment Strategy. International Journal of Innovative Computing, Information and Control, 5, 2169-2188.

[4] Cox, J.C., Ingersoll, J.E. and Ross, S.A. (1985) A Theory of the Term Structure of Interest Rates. Econometrica, 53, 385-407. https://doi.org/10.2307/1911242

[5] Privault, N. (2012) An. Elementary Introduction to Stochastic Interest Rate Modeling. 2nd Edition, Advanced Series on Statistical Sciences and Applied Probability, Vol. 16, World Scientific, Singapore.

[6] Brigo, D. and Mercurio, F. (2006) Interest Rate Models-Theory and Practice, with 
Smile, Inflation and Credit. 2nd Edition, Springer, Finance.

[7] Jeanblanc, M., Yor, M. and Chesney, M. (2009) Mathematical Methods for Financial Markets. Springer, Finance. https://doi.org/10.1007/978-1-84628-737-4 